\title{
Diagnóstico cardiaco basado en la probabilidad aplicado a pacientes con marcapasos
}

\section{Cardiac diagnosis based on probability applied to patients with pacemakers}

\author{
Javier Rodríguez, Signed Prieto, Catalina Correa, Pedro Bernal, \\ Sarith Vitery, luisa Álvarez, Natalia Aristizábal, Jorge Reynolds • \\ BogotÁ, D.C. (Colombia)
}

\section{Resumen}

Introducción: la teoría de la probabilidad permitió el desarrollo de una nueva metodología de ayuda diagnóstica del Holter. En este trabajo se aplicó este método al diagnóstico a pacientes con marcapasos.

Metodología: se seleccionaron 5 Holter normales y 10 de pacientes mayores de 21 años con implante de marcapasos. Se calculó la probabilidad del número de frecuencias obtenidas en los rangos definidos, respecto a la totalidad de frecuencias obtenidas en cada Holter, durante mínimo 21 horas, cuantificando el número de rangos en cada paciente, además de estimar la diferencia entre los rangos de los dos valores de frecuencia con mayor probabilidad y la suma de las probabilidades de dichos valores. Se evaluó la probabilidad del número total de latidos por hora a partir de rangos de 250 latidos, determinando la máxima probabilidad encontrada en cada Holter y determinando el diagnóstico físico matemático con base en los parámetros establecidos en el trabajo previo.

Resultados: las dinámicas con marcapasos presentaron para el parámetro de evaluación 1, un total de rangos de frecuencias que osciló entre 9 y 16, donde sólo un Holter presentó un valor superior a 14 , requiriendo su evaluación con los parámetros 2 y 3 , evidenciando que las dinámicas de pacientes con implante de marcapasos presentan siempre un comportamiento característico de enfermedad. Las dinámicas normales presentaron entre 16 y 21 rangos, donde sólo dos Holter presentaron valores menores o iguales a 17 , los cuales al ser evaluados con los parámetros restantes fueron diagnosticados como normales. El sobrelapamiento de las probabilidades de las frecuencias cardiacas evidencia que la variabilidad de la frecuencia cardiaca no diferencia fundamentalmente normalidad de enfermedad y evolución entre éstas.

Conclusión: la metodología con base en la probabilidad es adecuada para detectar el estado de anormalidad de la dinámica cardiaca de pacientes con marcapasos. (Acta Med Colomb 2012; 37: 183-191)

Palabras clave: Holter, probabilidad, marcapasos, diagnóstico.

\footnotetext{
Abstract

Introduction: the theory of probability allowed the development of a new diagnostic aid methodology of Holter. In this work we applied this method to diagnose patients with pacemakers.

Methods: we selected 5 normal Holter and 10 patients over 21 years with pacemaker implantation. We calculated the probability of the number of frequencies obtained in the ranges defined with respect to the totality of frequencies obtained in each Holter, during at least 21 hours, quantifying the number of ranges in each patient, in addition to estimating the difference between the most likely two frequency value ranges and the sum of the probabilities of these values. We assessed the likelihood of the total number of beats per hour from ranges of 250 beats, determining the maximum likelihood found in each Holter and determining the physical mathematical diagnosis based on parameters established on the previous work.
}

Instituciones: Grupo Insight • Línea de Profundización e Internado Especial: Teorías Físicas y Matemáticas Aplicadas a la Medicina - Universidad Militar Nueva Granada - Centro de Investigaciones de la Clínica del Country • Proyecto Nano Puente A-V.

Dr. Javier Rodríguez: Director del Grupo Insight. Director de la Línea de Profundización e Internado Especial: Física y Matemáticas Aplicadas a la Medicina, Universidad Militar Nueva Granada, Centro de Investigaciones Clínica del Country; Dra. Signed Prieto: Investigadora, Grupo Insight, Centro de Investigaciones Clínica del Country; Dra. Catalina Correa: Psicóloga, Investigadora Grupo Insight. Profesora de la Línea de Profundización e Internado Especial: Física y Matemáticas Aplicadas a la Medicina, Universidad Militar Nueva Granada, Centro de Investigaciones Clínica del Country; Dr. Pedro Bernal: Investigador Grupo Insight, Centro de Investigaciones Clínica del Country; Sarith Vitery: Interna Especial: Física y Matemáticas Aplicadas a la Medicina, Universidad Militar Nueva Granada, Centro de Investigaciones Clínica del Country; Dra. Luisa Álvarez: Psicóloga, Investigadora Grupo Insight, Centro de Investigaciones Clínica del Country; Natalia Aristizabal: Investigadora Grupo Insight, Centro de Investigaciones Clínica del Country; Dr. Jorge Reynolds: Ingeniero Electrónico, Director del Grupo Seguimiento Corazón Vía Satélite (SCVS). Bogotá D.C. (Colombia).

Correspondencia. Dr. Javier Rodríguez Velásquez. Bogotá D.C. (Colombia). E-mail: grupoinsight2025@yahoo.es Recibido: 18/XI/2011 Aceptado: 25/X/2012 
Results: the dynamics with pacemakers presented for 1evaluation parameter a total range of frequencies that ranged from 9 to 16 , where only one holter presented a value greater than 14, requiring evaluation with 2 and 3 parameters, showing that dynamics of patients with implanted pacemakers have always a characteristic disease behavior. Normal dynamics showed ranges between 16 and 21, with only two holter showing values less than or equal to 17 , which when evaluated with the remaining parameters were diagnosed as normal. The overlap of probabilities of the heart rates evidence that the heart rate variability does not differentiate essentially normality from disease, nor evolution among these.

Conclusion: the method based on the likelihood is suitable for detecting the abnormality state of heart dynamics of patients with pacemakers. (Acta Med Colomb 2012; 37: 183-191)

Keywords: Holter, probability, pacemakers, diagnosis.

\section{Introducción}

Uno de los mayores temas de interés en cardiología en la actualidad es el estudio de la variabilidad de la frecuencia cardiaca a partir de los cambios RR en el tiempo. Diferentes trabajos han evidenciado asociaciones entre la variabilidad RR y muerte súbita arrítmica (1), muerte por falla cardiaca (2), y eventos arrítmicos no fatales después de un infarto agudo de miocardio (3). Sin embargo, el carácter predictivo de estas variables no es suficiente para establecer diagnósticos de aplicación clínica, pues aunque la presencia de valores normales hace poco probable un evento patológico, un valor anormal no necesariamente implica que el paciente va a presentar una complicación cardiovascular. En este contexto se hace necesario el desarrollo de medidas matemáticas objetivas y reproducibles de carácter diagnóstico y de aplicación clínica para la evaluación de la dinámica cardiaca.

La teoría de sistemas dinámicos describe el estado y la evolución de los sistemas (4). Los sistemas dinámicos pueden ser representados mediante espacios abstractos denominados espacios de fase, obteniendo atractores cuya forma evidencia su carácter predecible o impredecible. En el caso de los sistemas impredecibles se obtienen atractores caóticos, cuya irregularidad puede ser caracterizada por medio de la geometría fractal $(5,6)$. En el estudio de la fisiología cardiaca a partir de la teoría de sistemas dinámicos ha sido posible desarrollar una concepción de normalidad/ enfermedad según la cual las dinámicas muy regulares así como las altamente aleatorias o irregulares están asociadas a enfermedad, en tanto que un comportamiento entre estos estados se asocia a normalidad, contradiciendo de este modo la concepción homeostática convencional, donde la normalidad se asocia a la regularidad (7). También se han desarrollado gran cantidad de investigaciones que buscan establecer parámetros de evaluación de la dinámica cardiaca de utilidad clínica. Entre ellos se cuentan medidas como exponentes de Lyapunov (8), dimensión de correlación D2 $(8,9)$, dimensión Hausdorff $(8,10)$, o dinámica simbólica para el análisis de la variabilidad de la dinámica cardiaca (11-13), entre otros. Con base en análisis fractales se han desarrollado mejores parámetros predictivos que los empleados convencionalmente en la clínica, para mortalidad de pacientes con infarto agudo de miocardio con fracción de eyección menor al 35\% (14). Sin embargo, estudios recientes indican que la aplicabilidad clínica de estas metodologías aún no ha podido ser establecida, pues presentan limitaciones de diversos tipos, que dificultan su aplicación clínica $(15,16)$.

La concepción de normalidad/enfermedad de la teoría de sistemas dinámicos ha sido base para el desarrollo de nuevas metodologías de evaluación en cardiología; por ejemplo, una reinterpretación de esta concepción y la aplicación de la ley de Zipf-Mandelbrot permitieron el desarrollo de una nueva metodología diagnóstica para la monitoría cardiaca fetal $(17,18)$. Así mismo se han desarrollado metodologías para la evaluación del Holter, obteniendo diferenciaciones matemáticas entre las dinámicas cardiacas agudas y un grupo de normales y crónicas $(19,20)$, y logrando diferenciar en un trabajo de tipo predictivo dinámicas cardiacas normales, crónicas y agudas, además de estados de evolución entre normalidad y enfermedad a partir de la probabilidad y las proporciones de la entropía (21); estas medidas predijeron la recuperación de un paciente de una intervención de ablación, al observar la disminución de un valor matemático que cuantifica las proporciones de miles que se encuentra por fuera de los límites de normalidad en el análisis de la dinámica cardiaca del pacientes durante tres días posteriores a dicha intervención. Los valores hallados fueron de $0.88,0.60$ y 0.41 , y su disminución progresiva da cuenta de su mejoría según predicciones de los parámetros fisicomatemáticos de la teoría, que también fue observada en la evaluación clínica convencional (21).

En esta línea de trabajo también se desarrolló una nueva metodología diagnóstica de evaluación del Holter con base en la teoría de la probabilidad, que puede ser usada como herramienta de aplicación clínica (22). Las leyes de la probabilidad fueron aplicadas a rangos de aparición de la frecuencia cardiaca y del número total de latidos cada hora, encontrando que la aplicación consecutiva de tres parámetros definidos permite la diferenciación entre normalidad y enfermedad; esta metodología fisicomatemática caracterizó de una forma objetiva y reproducible la dinámica cardiaca, con base en una autoorganización matemática que es aplicable a cualquier caso particular para individuos mayores de 20 años, y su efectividad diagnóstica es independiente 
de la edad, patología e intervenciones (22). Este trabajo evidencia que las medidas de variabilidad no permiten establecer diferencias diagnósticas aplicables para cada caso individual, pues tanto para normalidad como para enfermedad se encuentran valores de probabilidad de la frecuencia cardiaca en los diferentes rangos, comunes y también diferentes; y lo mismo ocurre al observar aisladamente los parámetros planteados en dicho trabajo; por el contrario, tanto éste como otros trabajos $(21,23)$ evidencian que sólo es posible establecer diferencias de carácter diagnóstico a nivel individual, no poblacional, mediante métodos que evalúen cuantitativamente la autoorganización del sistema, la cual no es observable con las medidas de variabilidad.

La teoría de la probabilidad fue desarrollada con el fin de cuantificar matemáticamente la posible ocurrencia de un evento en el futuro, tomando valores entre $0 \mathrm{y} 1$, donde 0 corresponde a imposibilidad de la presentación de un evento, y 1 corresponde a la total seguridad de que éste se presentará (24-26). La definición axiomática de la probabilidad corresponde a una función establecida sobre el espacio muestral S, que cumple tres condiciones: la probabilidad de cualquier evento es positiva, la probabilidad del espacio muestral $P(S)$ es igual a uno, y la probabilidad de la unión de eventos independientes entre sí corresponde a la suma de sus probabilidades (26).

El marcapasos es un dispositivo que ayuda al corazón a contraerse con un ritmo constante mediante estimulación eléctrica. Los marcapasos pueden ayudar a regular el ritmo del corazón en casos de frecuencia cardiaca lenta, rápida o irregular, o de bloqueo en el sistema de conducción eléctrica del corazón. La mayoría de los marcapasos puede detectar el ritmo cardiaco y apagarse cuando la velocidad de la frecuencia cardiaca alcanza un límite superior predeterminado, este tipo de marcapasos son llamados marcapasos a demanda, o pueden ser implantados marcapasos con una tasa fija de descarga los cuales son llamados asincrónicos (27). El uso del marcapasos ha permitido un tipo de tratamiento de intervención directa sobre enfermedades cardiacas que incluso hoy en día responden erráticamente a tratamientos como el farmacológico, controlando enfermedades que antes eran mortales y mejorando el pronóstico y tiempo de vida de los pacientes (28). El Holter permite detectar, reconocer, amplificar y registrar artefactos del marcapaso en un canal separado, de manera que puedan identificarse fallas en la captura, detección o salida del estímulo proveniente del marcapaso así como el número de ciclos estimulados y su porcentaje durante el día (29).

En este contexto, el propósito de este trabajo es aplicar la metodología de evaluación desarrollada con base en la teoría de la probabilidad al Holter de pacientes con implante de marcapasos y normales, con el fin de establecer la capacidad de la metodología para detectar el estado patológico en el caso específico de las dinámicas cardiacas reguladas por marcapasos, obteniendo una medida independiente de las medidas de variabilidad que evalúe objetivamente la auto-organización del sistema cardiaco en presencia de este dispositivo.

\section{Definiciones}

\section{Material y métodos}

Rango de frecuencia cardiaca: rango de 5 latidos/minuto en el que se ubica el valor de la frecuencia cardiaca (22).

Rango del número de latidos: rango de 250 latidos donde se halla el número de latidos de cada hora (22).

Probabilidad del rango: número de repeticiones de cada rango medido $\mathrm{N}_{\mathrm{R}}$, dividido entre el total de repeticiones de los rangos medidos $\mathrm{N}$ :

$P(A)=\frac{\text { Repeticiones de rango } r}{\text { Total de repeticiones de rangos medidos }}=\frac{N_{R}}{N}$
Ecuación 1

Se analizaron 5 Holter de individuos normales y 10 Holters de pacientes con edad igual o mayor a 21 años, con implante de marcapasos, 8 holters ambulatorios y 2 de un mismo paciente en unidad de cuidados coronarios durante dos días consecutivos. Los Holters y registros cardiacos continuos fueron realizados en la Fundación Cardio infantil, y sus diagnósticos desde los parámetros clínicos convencionales fueron establecidos por un cardiólogo experto. Las indicaciones y conclusiones del estudio de cada paciente se aprecian en la Tabla 1. Se tomaron pocos casos en principio para caracterizar matemáticamente el fenómeno, compararlo respecto a la normalidad, y evaluar si las dinámicas con marcapasos presentaban valores matemáticos asociados a estados patológicos.

Siguiendo la metodología desarrollada previamente (22), los valores máximos y mínimos de las frecuencias cardiacas cada hora durante mínimo 21 horas se catalogaron en rangos de cinco latidos por minuto (por ejemplo, el rango de 70 lat/ min incluye los valores de frecuencia entre 68 y 72 lat $/ \mathrm{min}$, mientras que el de 75 incluye los valores entre 73 y 77 lat/ $\mathrm{min})$, y se calculó la probabilidad del número de frecuencias obtenidas en cada rango respecto a la totalidad de frecuencias obtenidas en cada registro. Adicionalmente se contó el número de rangos que presentaban valores de probabilidad en cada paciente, la diferencia entre los rangos de los dos valores de frecuencia con mayor probabilidad y la suma de las probabilidades de dichos valores. Posteriormente se determinó la probabilidad del número total de latidos por hora cuantificada en rangos de 250 latidos, durante mínimo 21 horas, determinando la máxima probabilidad encontrada en cada Holter y se estableció el número de latidos mínimo y máximo. Los valores obtenidos fueron analizados de acuerdo con los tres parámetros establecidos para la obtención del diagnóstico matemático para cada dinámica (22), estableciendo el diagnóstico matemático de normalidad o anormalidad para cada caso. 
Este trabajo fue realizado cumpliendo con las normas científicas, técnicas y administrativas para la investigación en salud, de acuerdo con la resolución No. 008430 de 1993, y específicamente con el título 11 referente a la investigación en seres humanos, siendo catalogado en la categoría de investigación sin riesgo, pues se hacen cálculos sobre resultados de exámenes prescritos médicamente y que no afectan a los pacientes, respetando su anonimato.

\section{Resultados}

Se encontraron valores de las frecuencias cardiacas entre 45 y $150 \mathrm{lat} / \mathrm{min}$, para Holters con marcapasos y entre 45 y 165 para los normales. Los valores de la probabilidad encontrados para la frecuencia cardiaca de pacientes con marcapasos variaron entre 0 y 0,561 , los de Holters normales entre 0 y 0,242 (Tabla 2).

Al comparar entre normalidad y Holter con marcapasos, se evidenció que la distribución de las probabilidades de las frecuencias cardiacas presenta sobrelapamientos, con lo cual no es posible diferenciar normalidad de enfermedad mediante esta única observación. Este resultado evidencia que las medidas de variabilidad de la frecuencia cardiaca no permiten establecer diferencias a nivel diagnóstico.

El número de rangos encontrado para las frecuencias de los Holters de pacientes con implante de marcapasos osciló entre 9 y 16, encontrando que sólo un caso (No. 3) presentó un valor superior a 14 en el parámetro 1, por lo cual debió ser evaluado por los parámetros $2 \mathrm{a}, 2 \mathrm{~b}$ y 3 , con base en los cuales se determinó matemáticamente la enfermedad (22). Los Holters normales presentaron entre 16 y 21 rangos, de los cuales dos (No. 11 y 13) presentaron valores entre 14 y 17 , con lo cual requirieron la aplicación de los parámetros 2 y 3 , mediante los cuales se estableció su estado de normalidad.

Para establecer el parámetro 2a, se determinaron los valores de diferencia entre los rangos de las frecuencias más probables, los cuales se encontraron entre 5 y 25 para

Tabla 1. Edad, indicaciones y diagnóstico convencional de los Holter evaluados.

\begin{tabular}{|c|c|c|c|}
\hline \multirow[t]{2}{*}{ No. } & \multirow[t]{2}{*}{ Edad } & \multicolumn{2}{|l|}{ MARCAPASOS } \\
\hline & & Indicaciones & Conclusiones \\
\hline 1 & 43 & Control marcapasos & $\begin{array}{l}\text { Defecto por sobresensado ventricular que debe correlacionarse con los parámetros } \\
\text { de programación del dispositivo }\end{array}$ \\
\hline 2 & 62 & Portador de marcapasos, infarto hace 25 años & Marcapasos definitivo unicameral VVI-R normofuncionante \\
\hline 3 & 23 & Portador de marcapasos & Marcapasos definitivo unicameral VVIR normofuncionante \\
\hline 4 & 75 & Control marcapasos & 1 Extrasístole ventricular - 33 extrasístoles supraventriculares \\
\hline 5 & 65 & Arritmia & $\begin{array}{l}\text { Ritmo marcapasos bicameral DDD, que alterna con ritmo sinusal y conducción } \\
\text { AV e intraventricular normal. El dispositivo se observa normofuncionante con } \\
\text { adecuado sensado y captura auricular y ventricular. No se observan arritmias } \\
\text { supraventriculares ni ventriculares. El intervalo QTc es normal. No anotó síntomas } \\
\text { en el diario. Marcapaso definitivo bicameral normofuncionante. }\end{array}$ \\
\hline 6 & 81 & Control marcapasos & Marcapaso VVI-R normofuncionante \\
\hline 7 & 77 & Taquiarritmia & Ritmo de marcapaso de modo DDD \\
\hline 8 & 23 & Control & Marcapaso VVI-R normofuncionante \\
\hline 9 & 79 & $\begin{array}{l}\text { Paciente en UCC - Día } 1 \\
\text { Sindrome de bajo gasto cardiaco } \\
\quad \text { secundaría a ritmo idioventricular. } \\
\text { POP Primer día de PCI + } 3 \text { stent en CD. } \\
\text { IAM CEST inferior Killip clase I tipo } 1 \\
\text { no trombolizado. } \\
\text { Angina post infarto. } \\
\text { Cardiopatía isquémica FEVI confirmar. } \\
\text { Enfermedad coronaria de tres vasos. } \\
\text { HIA crónica. DM tipo } 2 \text {. }\end{array}$ & Marcapaso AAI 50X \\
\hline \multirow[t]{2}{*}{10} & 79 & $\begin{array}{l}\text { Paciente en UCC - Día } 2 \\
\text { Marcapaso AAI 50X. } \\
\text { Retiro de marcapasos transitorio }\end{array}$ & No síntomas cardiovasculares, evolución hacia la mejoría \\
\hline & & NORMALES & \\
\hline 11 & 25 & Estudio de síncope & Estudio dentro límites normales \\
\hline 12 & 71 & Taquicardia & Estudio dentro límites normales \\
\hline 13 & 58 & Estudio de síncope & Estudio dentro límites normales \\
\hline 14 & 31 & Bradicardia & Estudio dentro límites normales \\
\hline 15 & 76 & Palpitaciones & Estudio dentro límites normales \\
\hline
\end{tabular}


Tabla 2. Parámetro 2a. Distribuciones de probabilidad de frecuencias cardiacas de los Holter estudiados.

\begin{tabular}{|c|c|c|c|c|c|c|c|c|c|c|c|c|c|c|c|}
\hline \multirow{2}{*}{ Frecuencia } & \multicolumn{10}{|c|}{ Holter con marcapasos } & \multicolumn{5}{|c|}{ Holter Normales } \\
\hline & 1 & 2 & 3 & 4 & 5 & 6 & 7 & 8 & 9 & 10 & 11 & 12 & 13 & 14 & 15 \\
\hline 45 & 0 & 0 & 0 & 0 & 0 & 0 & 0 & 0 & 0.029 & 0 & 0 & 0 & 0.043 & 0.145 & 0 \\
\hline 50 & 0.106 & 0 & 0 & 0 & 0.015 & 0 & 0 & 0.238 & 0.043 & 0 & 0 & 0 & 0.130 & 0.164 & 0 \\
\hline 55 & 0.212 & 0 & 0 & 0 & 0.061 & 0 & 0.014 & 0.032 & 0.071 & 0.111 & 0.014 & 0.01 & 0.145 & 0.145 & 0 \\
\hline 60 & 0.258 & 0.413 & 0.015 & 0.030 & 0.242 & 0.561 & 0.528 & 0.317 & 0.129 & 0.167 & 0.116 & 0.104 & 0.159 & 0.055 & 0.030 \\
\hline 65 & 0.152 & 0.032 & 0.273 & 0.152 & 0.212 & 0.152 & 0.139 & 0.127 & 0.171 & 0.069 & 0.130 & 0.125 & 0.058 & 0.091 & 0.242 \\
\hline 70 & 0.076 & 0.127 & 0.045 & 0.152 & 0.152 & 0.091 & 0.125 & 0.111 & 0.100 & 0.083 & 0.145 & 0.087 & 0.145 & 0.018 & 0.091 \\
\hline 75 & 0.061 & 0.111 & 0 & 0.182 & 0.045 & 0.030 & 0.069 & 0.063 & 0.143 & 0.167 & 0.116 & 0.108 & 0.043 & 0.091 & 0.045 \\
\hline 80 & 0.061 & 0.095 & 0.030 & 0.212 & 0.076 & 0.045 & 0.056 & 0.016 & 0.214 & 0.208 & 0.087 & 0.13 & 0.029 & 0.036 & 0.015 \\
\hline 85 & 0.030 & 0.111 & 0.015 & 0.152 & 0.045 & 0.045 & 0.028 & 0.032 & 0.086 & 0.097 & 0.101 & 0.054 & 0.029 & 0.055 & 0.015 \\
\hline 90 & 0.015 & 0.032 & 0.106 & 0.045 & 0.061 & 0 & 0.028 & 0.016 & 0 & 0.056 & 0.029 & 0.072 & 0.029 & 0.018 & 0.076 \\
\hline 95 & 0.015 & 0.032 & 0.106 & 0.061 & 0.015 & 0.030 & 0.014 & 0 & 0.014 & 0 & 0.043 & 0.042 & 0.058 & 0.018 & 0.045 \\
\hline 100 & 0.015 & 0.032 & 0.061 & 0.015 & 0.061 & 0.015 & 0 & 0.016 & 0 & 0.014 & 0.087 & 0.029 & 0.014 & 0.036 & 0.030 \\
\hline 105 & 0 & 0.016 & 0.045 & 0 & 0 & 0.030 & 0 & 0.016 & 0 & 0.028 & 0.029 & 0.041 & 0.043 & 0.018 & 0.076 \\
\hline 110 & 0 & 0 & 0 & 0 & 0 & 0 & 0 & 0.016 & 0 & 0 & 0.014 & 0.029 & 0.029 & 0.018 & 0.015 \\
\hline 115 & 0 & 0 & 0.076 & 0 & 0.015 & 0 & 0 & 0 & 0 & 0 & 0.014 & 0.029 & 0.029 & 0.036 & 0.045 \\
\hline 120 & 0 & 0 & 0.076 & 0 & 0 & 0 & 0 & 0 & 0 & 0 & 0.014 & 0.054 & 0 & 0.018 & 0.030 \\
\hline 125 & 0 & 0 & 0.061 & 0 & 0 & 0 & 0 & 0 & 0 & 0 & 0.014 & 0.03 & 0 & 0 & 0 \\
\hline 130 & 0 & 0 & 0.045 & 0 & 0 & 0 & 0 & 0 & 0 & 0 & 0.043 & 0.006 & 0.014 & 0.018 & 0.015 \\
\hline 135 & 0 & 0 & 0.030 & 0 & 0 & 0 & 0 & 0 & 0 & 0 & 0 & 0.01 & 0 & 0 & 0.045 \\
\hline 140 & 0 & 0 & 0 & 0 & 0 & 0 & 0 & 0 & 0 & 0 & 0 & 0.04 & 0 & 0 & 0.045 \\
\hline 145 & 0 & 0 & 0 & 0 & 0 & 0 & 0 & 0 & 0 & 0 & 0 & 0 & 0 & 0.018 & 0.045 \\
\hline 150 & 0 & 0 & 0.015 & 0 & 0 & 0 & 0 & 0 & 0 & 0 & 0 & 0 & 0 & 0 & 0.015 \\
\hline 155 & 0 & 0 & 0 & 0 & 0 & 0 & 0 & 0 & 0 & 0 & 0 & 0 & 0 & 0 & 0.045 \\
\hline 160 & 0 & 0 & 0 & 0 & 0 & 0 & 0 & 0 & 0 & 0 & 0 & 0 & 0 & 0 & 0.015 \\
\hline 165 & 0 & 0 & 0 & 0 & 0 & 0 & 0 & 0 & 0 & 0 & 0 & 0 & 0 & 0 & 0.015 \\
\hline Rangos & 11 & 10 & 15 & 9 & 12 & 9 & 9 & 12 & 10 & 10 & 16 & 18 & 16 & 18 & 21 \\
\hline
\end{tabular}

los casos con marcapasos. Se encontró que sólo los Holter 3 y 9 del grupo de marcapasos presentaron valores iguales o superiores a 15 cumpliendo el parámetro 2a, lo que los cataloga dentro de la enfermedad. Los valores de los casos normales variaron entre 5 y 15 , donde sólo el Holter 14 presentó este último valor; sin embargo, por no cumplir con el parámetro 1, ya había sido diagnosticado matemáticamente como normal (22).

$\mathrm{Al}$ evaluar los totales de latidos por hora, se encontró que estas dinámicas tuvieron rangos entre 2000 y 6500, correspondientes a 18 rangos. Respecto al parámetro $2 \mathrm{~b}$, sólo el Holter 3, correspondiente al grupo de marcapasos, presentó un número de latidos superior a 6250 , mientras que siete Holter presentaron un número de latidos inferior a 3000, dos correspondientes al grupo de marcapasos (No. 9 y 10) y tres correspondientes al grupo de Holter normales (No. 13, 14 y 15).

Al evaluar conjuntamente los parámetros para establecer el diagnóstico fisicomatemático (22), se encontró que al comparar los casos con implantes de marcapasos entre casos normales se logra hacer una distinción entre las dinámicas cardiacas. En el caso de los Holter con implante de marcapasos se encontró que la dinámica siempre está diagnosticada matemáticamente como enferma, mientras que en los Holter normales siempre se obtiene un diagnóstico matemático de normalidad. 
Tabla 3. Distribución de probabilidades del número total de latidos por hora de cada Holter.

\begin{tabular}{|c|c|c|c|c|c|c|c|c|c|c|c|c|c|c|c|}
\hline \multirow{2}{*}{$\begin{array}{l}\text { Total } \\
\text { Latidos }\end{array}$} & \multicolumn{10}{|c|}{ Holter con marcapasos } & \multicolumn{5}{|c|}{ Holter normales } \\
\hline & 1 & 2 & 3 & 4 & 5 & 6 & 7 & 8 & 9 & 10 & 11 & 12 & 13 & 14 & 15 \\
\hline 2000 & 0 & 0 & 0 & 0 & 0 & 0 & 0 & 0 & 0.412 & 0.294 & 0 & 0 & 0 & 0 & 0.045 \\
\hline 2250 & 0 & 0 & 0 & 0 & 0 & 0 & 0 & 0 & 0.353 & 0.353 & 0 & 0 & 0 & 0.087 & 0.045 \\
\hline 2500 & 0 & 0 & 0 & 0 & 0 & 0 & 0 & 0 & 0.235 & 0.294 & 0 & 0 & 0 & 0.174 & 0 \\
\hline 2750 & 0 & 0 & 0.045 & 0.182 & 0 & 0 & 0.042 & 0 & 0 & 0 & 0 & 0 & 0.043 & 0.087 & 0 \\
\hline 3000 & 0 & 0 & 0.045 & 0.136 & 0 & 0 & 0 & 0.095 & 0 & 0.059 & 0 & 0 & 0.043 & 0.130 & 0.182 \\
\hline 3250 & 0.091 & 0 & 0 & 0.182 & 0.091 & 0 & 0.708 & 0.143 & 0 & 0 & 0 & 0.043 & 0.174 & 0.261 & 0.045 \\
\hline 3500 & 0.455 & 0.286 & 0 & 0.136 & 0.136 & 0 & 0.167 & 0 & 0 & 0 & 0 & 0 & 0.130 & 0.043 & 0.409 \\
\hline 3750 & 0.136 & 0.333 & 0 & 0.182 & 0.364 & 0.727 & 0 & 0.190 & 0 & 0 & 0 & 0.219 & 0.218 & 0.087 & 0.182 \\
\hline 4000 & 0.318 & 0.286 & 0.045 & 0.182 & 0.227 & 0.182 & 0 & 0 & 0 & 0 & 0.261 & 0.130 & 0.174 & 0.087 & 0.045 \\
\hline 4250 & 0 & 0.095 & 0.045 & 0 & 0.091 & 0.091 & 0.042 & 0.048 & 0 & 0 & 0.043 & 0 & 0.043 & 0.043 & 0.045 \\
\hline 4500 & 0 & 0 & 0.182 & 0 & 0.045 & 0 & 0.042 & 0.238 & 0 & 0 & 0.130 & 0.176 & 0.043 & 0 & 0 \\
\hline 4750 & 0 & 0 & 0.045 & 0 & 0.045 & 0 & 0 & 0.048 & 0 & 0 & 0.130 & 0.130 & 0 & 0 & 0 \\
\hline 5000 & 0 & 0 & 0.045 & 0 & 0 & 0 & 0 & 0.095 & 0 & 0 & 0.261 & 0.130 & 0.043 & 0 & 0 \\
\hline 5250 & 0 & 0 & 0.091 & 0 & 0 & 0 & 0 & 0.048 & 0 & 0 & 0.087 & 0.043 & 0.086 & 0 & 0 \\
\hline 5500 & 0 & 0 & 0.045 & 0 & 0 & 0 & 0 & 0 & 0 & 0 & 0.043 & 0.043 & 0 & 0 & 0 \\
\hline 5750 & 0 & 0 & 0.136 & 0 & 0 & 0 & 0 & 0 & 0 & 0 & 0.043 & 0.043 & 0 & 0 & 0 \\
\hline 6000 & 0 & 0 & 0.182 & 0 & 0 & 0 & 0 & 0.095 & 0 & 0 & 0 & 0.043 & 0 & 0 & 0 \\
\hline 6250 & 0 & 0 & 0.045 & 0 & 0 & 0 & 0 & 0 & 0 & 0 & 0 & 0 & 0 & 0 & 0 \\
\hline 6500 & 0 & 0 & 0.045 & 0 & 0 & 0 & 0 & 0 & 0 & 0 & 0 & 0 & 0 & 0 & 0 \\
\hline
\end{tabular}

Tabla 4. Evaluación de los parámetros $1,2 \mathrm{2a}, \mathbf{2 b}$ y 3 . Rangos: Rangos de frecuencia cardiaca media. Diferencia: diferencia entre los dos rangos de frecuencia con mayor probabilidad, máxima probabilidad de número de latidos. $>\mathbf{6 2 5 0}$ : número de veces en que se presenta un número de latidos /hora superior a 6250. <3000: número de veces en que se presenta un número de latidos /hora superior a 6250. Suma: suma de las dos probabilidades de frecuencias con mayor valor.

\begin{tabular}{|c|c|c|c|c|c|c|}
\hline Paciente & Rangos & Diferencia & $\begin{array}{c}\text { Máx Prob } \\
\text { No. Lat }\end{array}$ & $>\mathbf{6 2 5 0}$ & $<\mathbf{3 0 0 0}$ & Suma \\
\hline $\mathbf{1}$ & 11 & 5 & 0.455 & & & 0.470 \\
\hline $\mathbf{2}$ & 10 & 10 & 0.333 & & & 0.540 \\
\hline $\mathbf{3}$ & 15 & 25 & 0.182 & $\mathrm{X}$ & $\mathrm{X}$ & 0.379 \\
\hline $\mathbf{4}$ & 9 & 5 & 0.182 & & $\mathrm{X}$ & 0.394 \\
\hline $\mathbf{5}$ & 12 & 5 & 0.364 & & & 0.454 \\
\hline $\mathbf{6}$ & 9 & 5 & 0.727 & & & 0.713 \\
\hline $\mathbf{7}$ & 9 & 5 & 0.708 & & & 0.667 \\
\hline $\mathbf{8}$ & 12 & 10 & 0.238 & & & 0.555 \\
\hline $\mathbf{9}$ & 10 & 15 & 0.412 & & $\mathrm{X}$ & 0.385 \\
\hline $\mathbf{1 0}$ & 10 & 5 & 0.353 & & $\mathrm{X}$ & 0.375 \\
\hline $\mathbf{1 1}$ & 16 & 5 & 0.261 & & & 0.275 \\
\hline $\mathbf{1 2}$ & 18 & 5 & 0.219 & & & 0.275 \\
\hline $\mathbf{1 3}$ & 16 & 5 & 0.218 & & $\mathrm{X}$ & 0.304 \\
\hline $\mathbf{1 4}$ & 18 & 15 & 0.261 & & $\mathrm{X}$ & 0.146 \\
\hline $\mathbf{1 5}$ & 21 & 10 & 0.409 & & $\mathrm{X}$ & 0.167 \\
\hline
\end{tabular}

\section{Discusión}

Este es el primer trabajo en el que se hace una aplicación de la metodología desarrollada para evaluación del Holter con base en la teoría de la probabilidad, a pacientes normales y con implante de marcapasos. Al caracterizar la dinámica cardiaca de estos pacientes y compararla con los parámetros establecidos para normalidad y enfermedad se encontró que la dinámica cardiaca de pacientes con marcapasos presenta un comportamiento característico de enfermedad, y se verificó la aplicabilidad de la metodología a cualquier caso particular con implante de marcapasos, independientemente de la patología, intervenciones previas y de la edad para individuos mayores de 21 años, por tratarse de una metodología que establece matemáticamente el estado de normalidad o enfermedad independientemente de las causas. En contraposición a los trabajos basados en medidas de variabilidad, que no pueden diferenciar qué es normal o anormal en medicina, en este trabajo se confirma que es posible detectar anormalidad con medidas probabilistas de la auto organización del sistema. En este sentido este trabajo es un avance al mostrar a la comunidad cardiológica la insuficiencia del concepto de variabilidad de la dinámica cardiaca para diagnosticar a nivel clínico, como en trabajos previamente realizados $(21,31)$ y 
presentar alternativas metodológicas que permitan evaluar la auto-organización del sistema. Sin embargo es necesario desarrollar trabajos más específicos para establecer la capacidad de esta metodología para evaluar el funcionamiento del marcapasos a nivel clínico.

La concepción homeostática tradicional asume la periodicidad del ritmo cardiaco como característica de normalidad; sin embargo, trabajos realizados desde la concepción de los sistemas dinámicos por Goldberger y cols. (7) han demostrado que el estado de salud está asociado a un comportamiento intermedio entre el comportamiento altamente irregular y la periodicidad. El hecho de que el dispositivo cardiaco es programado para mantener un comportamiento cardiaco regular, con base en la concepción tradicional, explica por qué al evaluar la dinámica cardiaca de pacientes con marcapasos se encuentra un diagnóstico de enfermedad. Es importante resaltar que aunque una dinámica aguda puede evolucionar en el tiempo y reorganizarse para tener una dinámica funcional, nunca volverá a ser una dinámica normal. Del mismo modo, una dinámica regulada con un marcapasos tampoco podrá presentar valores de normalidad, pues un corazón que ha sufrido una patología tan severa como para requerir un marcapasos se caracteriza por una autoorganización de su dinámica que puede permitirle una funcionalidad, pero nunca podrá tener nuevamente un funcionamiento completamente normal. Sin embargo, al tratarse con valores numéricos, es posible establecer comparaciones para ver variaciones de la dinámica en relación con intervenciones de carácter quirúrgico o farmacológico, permitiendo así un seguimiento más efectivo de los pacientes.

El Holter es un examen de amplia utilidad clínica, siendo empleado como herramienta de apoyo diagnóstico de alteraciones que implican la toma de decisión de implante de marcapasos y en la valoración del dispositivo (29); el Holter se ha evaluado comúnmente en la práctica clínica a partir de observaciones realizadas con base en estudios estadísticos, que por su propio método impiden efectuar afirmaciones sobre casos particulares. En contraposición, teorías físicas y matemáticas como la teoría de sistemas dinámicos, la geometría fractal, la teoría de la probabilidad o la entropía han permitido el desarrollo de metodologías objetivas, reproducibles y de aplicación a la práctica clínica para la evaluación del Holter (19-23). Recientemente, se desarrolló una metodología que permitió la diferenciación de enfermedad aguda de enfermedad crónica y normalidad, a partir de la comparación del número de espacios ocupados por el atractor cardiaco en el espacio de Box Counting (19) y cuya aplicabilidad fue confirmada estadísticamente en un estudio posterior (20). Del mismo modo, el trabajo desarrollado con base en las proporciones de la entropía del atractor, permite diferenciar normalidad, enfermedad aguda y enfermedad crónica, así como la evolución entre estos estados $(21,23)$.

En este trabajo sólo se toman pocos pacientes, el interés del estudio es caracterizar la dinámica cardiaca regulada por marcapasos y comparada con normalidad, con base en medidas de probabilidad independientes de las medidas de variabilidad. En este sentido se sigue la forma de pensamiento inductivo de la física teórica, donde se busca establecer características universales con base en pocos casos característicos, que permitan establecer afirmaciones de carácter predictivo. El primer paso para lograr una inducción de este tipo es establecer caracterizaciones matemáticas del fenómeno por estudiar, como se hace en este caso con las dinámicas cardiacas asociadas a marcapasos y normales. Tanto para el proceso de caracterización como para la inducción sólo se necesitan pocos casos, en contraposición con los trabajos basados en metodologías estadísticas, razón por la cual en este trabajo sólo se escogieron 10 Holter con marcapasos y cinco normales. Sin embargo, después de este proceso, es posible realizar análisis estadísticos para confirmar la aplicabilidad de las metodologías obtenidas de acuerdo con los criterios epidemiológicos. Recientemente se realizó un trabajo de este tipo, en el que se aplicó la metodología evaluada en el presente trabajo a 15 pacientes normales y 100 con diferentes patologías cardiacas, obteniéndose valores de sensibilidad y especificidad de $100 \%$ y $73.3 \%$ respectivamente, así como un coeficiente Kappa de 0.86, al ser comparadas con el diagnóstico convencional (30).

Este tipo de razonamiento inductivo ha sido aplicado a otros trabajos en cardiología logrando predicciones de aplicación clínica. El carácter predictivo de estas metodologías hace referencia a su capacidad para encontrar cambios de valores matemáticos en el tiempo, que dan cuenta de una evolución a estados de mayor o menor gravedad, no a lo que desde la medicina tradicional y la epidemiologia se concibe como "pronóstico". Por ejemplo, la metodología que diferencia normalidad y enfermedad crónica de enfermedad aguda, evidencia que una disminución de la ocupación espacial del atractor se asocia a una evolución a estados más agudos (20). Del mismo modo, los parámetros asociados a la evaluación el número de latidos de la metodología que se aplica en el presente manuscrito, fueron aplicados para la evaluación de pacientes arrítmicos, encontrando resultados que sugieren que estos parámetros pueden detectar dinámicas con alteraciones leves o en evolución a la enfermedad, no diagnosticadas de acuerdo con los parámetros convencionales (31). Así mismo, el trabajo desarrollado por Rodríguez con base en las medidas de proporciones de la entropía (21) ha permitido establecer medidas que confirman el carácter predictivo de esta última metodología diagnóstica en un estudio realizado en pacientes en la unidad de cuidados coronarios, en el que se evaluó de manera objetiva y cuantitativa la evolución de pacientes, evidenciando la aplicabilidad en la valoración de intervenciones quirúrgicas y/o farmacológicas a partir de las distancias de los valores encontrados respecto al límite de normalidad, al detectar dinámicas cuyas medidas matemáticas evolucionaban a estados de mayor gravedad aun en ausencia de síntomas clínicos, y que desembocaron en IAM (23). Estos y otros trabajos, al proveer cuantificaciones no 
sólo de normalidad y enfermedad, sino también de evolución, pueden ser de gran utilidad para la programación de marcapasos y la evaluación efectuada en el seguimiento de los pacientes con implante $(21,23,32)$.

Este trabajo sigue una línea de investigación en la cual se busca establecer la capacidad de la metodología propuesta para detectar anormalidad ante diferentes fenómenos, tales como arritmias (31), o en este caso dinámicas reguladas por marcapasos. Sin embargo, para el establecimiento de medidas más específicas, tanto del nivel de gravedad y su tendencia a estados agudos, como de las variaciones respecto a características específicas del marcapasos, por ejemplo, es necesario hacer uso de metodologías más potentes, tales como la que se basa en las medidas de las proporciones de la entropía o la ley del caos cardiaco $(21,23,32)$ y que han mostrado ser más específicas y con mayor capacidad predictiva.

La perspectiva teórica que sustenta la presente investigación sigue la forma de razonamiento acausal de las teorías de la física moderna, como la teoría del caos, la mecánica estadística y la mecánica cuántica, donde lo fundamental es establecer órdenes matemáticos subyacentes independientemente de análisis causales. Esta perspectiva teórica ha dado lugar a múltiples resultados diagnósticos de aplicación clínica, tanto en cardiología como en otros campos de la medicina. En el primer caso se han logrado resultados tales como una ley matemática de los sistemas dinámicos cardiacos, que permite establecer la totalidad de dinámicas cardiacas tanto de normalidad como de enfermedad y de evolución, y que puede ser aplicada al análisis de marcapasos para posibles futuras aplicaciones clínicas (32); así como una generalización mediante la cual se estableció la totalidad de posibles arterias normales y restenosadas en modelos experimentales de restenosis (33); también se han realizado diagnósticos fractales de la ramificación coronaria izquierda evaluada en angiografías (34), y del ventrículo izquierdo en ventriculogramas (35), con los cuales se logran evaluaciones simultáneas de la morfología y la fisiología cardiaca de aplicabilidad clínica. En el caso de áreas de la medicina diferentes a la cardiología, se han logrado predicciones y diagnósticos en campos como la inmunología (36), biología molecular (37), predicción de epidemias (38) y el diagnostico fisicomatemático de la morfofisiología celular en cáncer de cuello uterino $(39,40)$.

\section{Agradecimientos}

A la Fundación Cardio Infantil, en especial al Dr. Gabriel Salazar y al Dr. Daniel Isaza, por su apoyo constante a las investigaciones del grupo Insight.

A la Universidad Militar Nueva Granada, especialmente al fondo de Investigaciones, que financió el proyecto MED 842, del cual este trabajo constituye uno de sus resultados. Agradecemos especialmente a la Dra. Martha Bahamón, Vicerrectora Académica, al Dr. José Ricardo Cure Hakim, vicedecano de investigaciones, al Dr. Henry Acuña, Jefe de la división de Investigación Científica, a la Dra. Clara Benavides, Directora del Programa de Medicina, y a la Dra. Esperanza Fajardo, Directora del Centro de Investigaciones.

A todo el personal del Centro de Investigaciones de la Clínica del Country, especialmente a la Dra. Adriana Lizbeth Ortiz, epidemióloga del Centro,y al Dr. Andrés Rodríguez, director del Centro de Investigaciones y subdirector médico de la institución, por su apoyo a nuestras investigaciones.

\section{Fuentes de Financiación}

Este artículo es resultado del proyecto MED 842, financiado por el Fondo de investigaciones de la Universidad Militar Nueva Granada.

\section{Conflictos de interés}

Los autores declaran no tener ningún conflicto de interés.

\section{Referencias}

1. Barron H, Viskin S. Autonomic markers and prediction of cardiac death after myocardial infarction. Lancet 1998; 351: 461-2.

2. Nolan J, Batin PD, Andrews R et al. Prospective study of heart rate variability and mortality in chronic heart failure: results of the United Kingdom Heart Failure Evaluation and Assessment of Risk Trial (UK - heart). Circulation 1998; 98: $1510-6$

3. Wolf M, Varigos G, Hunt D, Sluman J. Sinus arrhythmia in acute myocardial infarction. Med J Aus 1978; 2: 52-3

4. Devaney R. A first course in chaotic dynamical systems theory and experiments. Reading Mass: Addison- Wesley 1992.

5. Peitgen H, Jurgens $\mathbf{H}$, Saupe $\mathbf{D}$. Chaos and fractals; New frontiers of science. New York. Springer-Verlag 1992

6. Peitgen H. Strange attractors, the locus of chaos. En: Peitgen H, Jurgens H, Saupe D. Chaos and Fractals; New Frontiers of Science. New York. Springer-Verlag 1992. p. 655-768.

7. Goldberger A, Amaral L, Hausdorff JM, Ivanov P, PengCh, Stanley HE. "Fractal dynamics in physiology: alterations with disease and aging". PNAS 2002; 99: 2466-2472.

8. Eckmann JP, Ruelle D. Ergodic theory of chaos and strange attractors. Rew Mod Physics 1985; 7: 617-56.

9. Grassberger P, Procaccia I. Measuring the strangeness of strange attractors. Physica 1983; 9: 189-208.

10. Babyloyantz A, Destexhe A. Is the normal heart a periodic oscillator? Bio Cybern 1988; 58: 203-11.

11. Kurths J, Voss A, Saparin P, Witt A, Kleiner H. J. \& Wessel N. Quantitative analysis of heart rate variability. Chaos 1995; 5: 88-94. (doi:10.1063/1.166090).

12. Guzzetti, S. et al. Symbolic dynamics of heart rate variability: a probe to investigate cardiac autonomic modulation. Circulation 2005 (112): 465-470. (doi:10.1161/CIRCULATIONAHA.104.518449).

13. Maestri R, Pinna G.D, Balocchi R, D'Addio G, Ferrario M, Porta A, Sass R, Signorini M. G. \& La Rovere M. T. 2006 Clinical correlates of non-linear indices of heart rate variability in chronic heart failure patients. Biomed. Tech. (Berl.) 2006; 51: 220-223.

14. Huikuri HV, Makikallio T, Peng CK, et al. Fractal correlation properties of $\mathrm{R}-\mathrm{R}$ interval dynamics and mortality in patients with depressed left ventricular function after and acute myocardial infarction. Circulation 2000; 101: 47-53.

15. Juha S. Perkio“Ma“ Ki, Timo H. Ma“ Kikallio, Huikuri H. Fractal and complexity measures of heart rate variability. Clin Exp Hypertens 2005; 2(3): 149-58.

16. Voss A, Schulz S, Schroeder R, Baumert M, Cminal P. Methods derived from nonlinear dynamics for analysing heart rate variability. Phil Trans $R$ Soc A 2009; 367: 277-296.

17. Rodríguez J, Prieto S, Ortiz L, Bautista A, Bernal P, Avilán N. Diagnóstico Matemático de la Monitoria Fetal aplicando la ley de ZipfMandelbrot. Rev Fac Med Univ Nac Colomb 2006; 54(2): 96-107.

18. Rodríguez J. Dynamical systems theory and ZIPF - Mandelbrot Law applied to the development of a fetal monitoring diagnostic methodology. XVIII FIGO World Congress Of Gynecology And Obstetric. Kuala Lumpur, Malaysia. November 2006

19. Rodríguez J, Prieto S, Avilán N, Correa C, Bernal P, Ortiz L, et al. Nueva metodología física y matemática de evaluación del Holter. Rev Colomb Cardiol 2008; 15: 50-54.

20. Rodríguez J, Prieto S, Bernal P, Soracipa Y, Salazar G, Izasa D, et al. Nueva metodología diagnóstica de la dinámica geométrica cardiaca. Dinámica cardiaca caótica del Holter. Rev Acad Colomb Cienc 2011; 35(134): 5-12.

21. Rodríguez J. Entropía Proporcional De Los Sistemas Dinámicos Cardiacos: Predicciones físicas y matemáticas de la dinámica cardiaca de aplicación clínica. Rev Colomb Cardiol 2010; 17: 115-129.

22. Rodríguez J, Correa C, Ortiz L, Prieto S, Bernal P, Ayala J. Evaluación matemática de la dinámica cardiaca con la teoría de la probabilidad. Rev Mex Cardiol 2009; 20(4): 183-9.

23. Rodríguez J, Prieto S, Bernal P, Isaza D, Salazar G, Correa C, et al. Entropía proporcional aplicada a la evolución de la dinámica cardiaca. Predicciones de aplicación clínica. En: La emergencia de los enfoques de la complejidad en 
América Latina. Argentina: Comunidad del Pensamiento Complejo. En Prensa.

24. Laplace P. Ensayo filosófico sobre las probabilidades. Barcelona: Altaya; 1995.

25. Feynman R, Leighton R, Sands M. Probabilidad. En: Feynman R, Leighton R, Sands M. Física. Vol. 1. Wilmington: Addison-Wesley Iberoamericana, S. A. 1964. p. 6-1, 6-16.

26. Mood A, Graybill F, Boes D. Introduction to the theory of statistics. 3a Ed Singapore: Mc. Graw-Hill; 1974.

27. Myerburg RJ. Implantablecardioverter-defibrillators after myocardial infarction. N Engl J Med 2008; 359(21): 2245-53.

28. Reynolds Pombo J. Historia de Los Marcapasos en Colombia. Rev Colomb Cardiol 1985; 1 .

29. Negrete Salcedo A, Gil Roncancio E. Valoración de la función de marcapasos por monitoría electrocardiográfica ambulatoria de 24 horas (Holter). Cabrales M, Vanegas D. Manual de métodos diagnósticos en electrofisiología cardiovascular. Bogotá. Sociedad Colombiana de Cardiología y Cirugía Cardiovascular 2006. p 49-65.

30. Rodríguez J, Correa C, Prieto S, Bernal P, Forero G, Salazar G, et al. Confirmación del método de ayuda diagnóstica de la dinámica cardiaca de aplicación clínica desarrollado con base en la teoría de la probabilidad. Rev Fac Med 2011; 19(2): 167-177.

31. Rodríguez J, Álvarez L, Tapia D, López F, Cardona M, Mora J, et al. Evaluación de la dinámica cardiaca de pacientes con arritmia con base en la Teoría de la Probabilidad. Medicina 2012; 1(34)96: 7-16.

32. Rodríguez J. Mathematical law of chaotic cardiac dynamic: Predictions of clinic application. J Med Med Sci 2011; 2(8): 1050-1059.
33. Rodríguez J, Prieto S, Correa C, Bernal P, Puerta G, Vitery S, et al Theoretical generalization of normal and sick coronary arteries with fractal dimensions and the arterial intrinsic mathematical harmony. BMC Medical Physics 2010; 10: 1

34. Rodríguez J, Prieto S, Correa C, Bernal P, Tapia D, Álvarez L, et al. Diagnóstico fractal de disfunción cardíaca severa. Dinámica fractal de la ramificación coronaria izquierda. Rev Colomb Cardiol 2012. En prensa.

35. Rodríguez J, Prieto S, Correa C, Bernal P, Álvarez L, Forero G, et al Diagnóstico fractal del ventriculograma cardiaco izquierdo. Geometría fractal del ventriculograma durante la dinámica cardiaca. Rev Colomb Cardiol 2012 19(1): $18-24$

36. Rodríguez J. Teoría de unión al HLA clase II teorías de Probabilidad Combinatoria y Entropía aplicadas a secuencias peptídicas. Inmunología 2008; 27(4): 151-166.

37. Rodríguez J, Bernal P, Prieto S, Correa C. Teoría de péptidos de alta unión de malaria al glóbulo rojo. Predicciones teóricas de nuevos péptidos de unión y mutaciones teóricas predictivas de aminoácidos críticos. Inmunología 2010; 29(1): 7-19.

38. Rodríguez J. Método para la predicción de la dinámica temporal de la malaria en los municipios de Colombia. Rev Panam Salud Pública 2010; 27(3): 211-218.

39. Rodríguez J. Nuevo método fractal de ayuda diagnóstica para células preneoplásicas del epitelio escamoso cervical. Rev U.D.C.A Act \& Div Cient 2011; 14(1): $15-22$

40. Rodríguez J, Prieto S, Correa C, Posso H, Bernal P, Puerta G, et al. Generalización Fractal de Células Preneoplásicas y Cancerígenas del Epitelio Escamoso Cervical. Una Nueva Metodología de Aplicación Clínica. Rev Fac Med 2010 18(2): 173-181. 